drop with steppage gait and was prescribed an articulating left ankle foot orthosis set in slight plantar flexion to improve knee stability.

Discussion: Preservation of hip flexion is seen in femoral nerve injuries below the inguinal ligament after the branch to psoas major. The patient was placed in lithotomy positioning during surgery with hips flexed, abducted, and externally rotated putting the femoral nerve in a potentially unfavorable position sharply angulated beneath the inguinal ligament. Blood is supplied to the femoral nerve via iliolumbar artery, deep circumflex iliac artery (in the inguinal region) and lateral circumflex artery. The right deep circumflex artery has been found in cadaveric studies to give more arterial branches to the nerve than the left; hence the left femoral nerve may be more vulnerable to ischemic injury and explain why only the left femoral nerve was compromised in this patient.

Conclusions: Unilateral knee extension weakness with preservation of hip flexion is an unusual presentation of iatrogenic femoral nerve injury which may be explained by lithotomy positioning and predilection for left sided involvement due to variable blood supply to the nerve. An additional caveat to this case was a concomitant common peroneal nerve injury posing challenges in both diagnosis and treatment.

Level of Evidence: Level $\mathrm{V}$

\section{Poster 360:}

Preliminary Study on the Mechanism of Repetition

Recovery in a Chinese Aphasia Patient by Combining

Resting-State fMRI and DTI: A Case Report

Wang Hong, MD (Guangzhou, China)

Disclosures: Wang Hong: I Have No Relevant Financial Relationships To Disclose

Case/Program Description: Case Description: This patient received a 4-months traditional speech therapy (ST) and achieved good speech improvement. Before and after the 4 months' ST, Aphasia Battery of Chinese $(A B C)$, which is a modified Western Aphasia Battery (WAB) adapted to Chinese culture, was applied to evaluate the patient's speech function. The r-fMRI and diffusion tensor imaging study (DTI) were performed with a 3.0T MR system. The fractional anisotropy (FA) was calculated with the Functool 9.4.05a software to approach the integrity of AF. The correlation coefficients for functional connection (FC) strength between Broca area (BA) and Wernicke area (WA) was determined with software DPARSF and REST.

Setting: An academic hospital.

Results: In the two sub-items of repetition, this patient demonstrated remarkable improvement in the word repetition (WR), but limited improvement in the sentence repetition (SR). The correlation coefficients between BA and WA showed an increase in left hemisphere, but a slight decrease in right hemisphere. The FA value in AF was found to have mild increase bilaterally.

Discussion: In this patient, both the structural connection (SC) repair of $A F$ revealed by DTI and the increased FC between BA and WA revealed by $r$ - $f M R I$ are consistent with his WR recovery. The decrease of the FC between BA and WA in right hemisphere suggested the down-regulation of the inhibition from the non-dominant hemisphere may also play a role in WR recovery. The relative limited recovery of SR may be due to the insufficient recovery of $\mathrm{AF}$.

Conclusions: Both FC and SC between BA and WA may contribute to WR recovery. However, SR recovery requires more complicated nerve routes and more solid SC recovery, and possibly involving wide brain areas.

Level of Evidence: Level V
Poster 361:

Psychogenic Polydipsia after Pediatric Severe Traumatic Brain Injury: A Case Report

Philip Chen, MD (University of Michigan, Ann Arbor, MI, United States), Ryan Stork, MD

Disclosures: Philip Chen, MD: I Have No Relevant Financial Relationships To Disclose

Case/Program Description: A 16-year-old male with severe traumatic brain injury (TBI) after a motor vehicle collision. During the patient's acute rehab stay, the patient and mother described an insatiable thirst. Oral fluid intake and urine outputs ranged from 3 to 7 liters per day. There was an initial clinical concern for diabetes insipidus (DI). Sodium remained normal throughout his admission. Serum osmolality remained in normal limits. Urine osmolality was low. Patient was unable to complete a water deprivation study during the inpatient stay due to profound thirst. After discharge, his polydipsia continued, including reports of drinking 5 gallons of milk per week.

Setting: Tertiary care pediatric hospital.

Results: A water deprivation test was performed as an outpatient confirming appropriate concentration of urine and excluding the diagnosis of DI. 6 months after discharge, family noted his thirst was improving, though still increased compared to baseline. A nutritionist assisted with behavioral management, as he also had hyperphagia causing significant weight gain.

Discussion: Sodium and fluid balance dysfunctions are commonly reported following severe TBI, including cerebral salt wasting, diabetes insipidus, and the syndrome of inappropriate ADH secretion. Psychogenic polydipsia, on the other hand, is a very rare complication after $\mathrm{TBI}$, being described in only two prior case reports to our knowledge, and none previously in a pediatric patient. Diabetes insipidus and psychogenic polydipsia have similar clinical presentations, but it is important to distinguish the two because the treatments are vastly different, and mistreatment can lead to serious consequences, including hyponatremia or hypernatremia. A water deprivation study should easily establish the correct diagnosis.

Conclusions: Psychogenic polydipsia is a rare complication of TBI. It is important to be aware of given the similarity in clinical presentation to diabetes insipidus, which is a more common complication after TBI.

Level of Evidence: Level V

\section{Poster 362:}

Nivolumab-Induced Concurrent Guillain-Barre

Syndrome and Myasthenia Gravis in a Patient with Metastatic Renal Cell Carcinoma: A Case Report

Isabel M. Rutzen-Lopez (UT MD Anderson Cancer Center), Jack B. Fu, $M D, F A A P M R$, Jose E. Arias-Berrios, MD

Disclosures: Isabel Rutzen-Lopez: I Have No Relevant Financial Relationships To Disclose

Case/Program Description: A 67-year-old man with metastatic renal cell carcinoma who had recently received immunomodulation therapy with Nivolumab, presented to the Emergency Center reporting 3 weeks of progressive diplopia, dysphagia, dysarthria, generalized weakness, and urinary and fecal incontinence. On admission, the patient developed rapidly progressive respiratory failure requiring endotracheal intubation. The patient was initially treated with high dose steroids and plasmapheresis for suspected immunotherapy-related Myasthenia Gravis. Extensive workup was performed, including brain imaging, EMG, lumbar puncture, and blood tests. Electrodiagnostic and lumbar puncture findings favored diagnosis of Miller Fisher variant of Guillain Barre Syndrome (GBS). Further workup revealed abnormal AchR antibody 